\title{
The competition and cooperation between hotel enterprise and OTA
}

\author{
Qiang $\mathrm{Yu}^{1, \mathrm{a}}$, Zhaohui Wang ${ }^{1, \mathrm{~b}}$ \\ ${ }^{1}$ Harbin University Harbin China \\ ayuqiang0708@163.com, bwzh7411@126.com
}

Keywords: Hotel enterprises, OTA, Competition and cooperation.

\begin{abstract}
Under the background of the Internet era, OTA achieved the counterattack on traditional tourism industry by means of information technology. Owing to the tremendous advantages of OTA network channels and consumer experience innovation, the traditional tourism industry is competing with OTA to achieve win-win cooperation by sharing business profits and market size. The hotel industry is one of the earliest branches of the tourism industry to seek win-win cooperation with OTA. However, in recent years, fierce competition in the OTA industry has undergone tremendous changes in the industry, the emergence of industry oligarchy, making OTA bargaining advantage more obvious. At the same time, after nearly 10 years of dormancy, hotel enterprises under the guidance of information technology continue to achieve industry innovation and industrial upgrading, the hotel enterprise information technology innovation capability continues to strengthen, This paper will discuss and analyze how hotel enterprises can exert their innovative advantages to compete and cooperate with OTA in the new round of competition.
\end{abstract}

\section{酒店企业与OTA的竞争与竞合}

\author{
于强1, a 王朝辉 $1, b$, \\ 1哈尔滨学院, 哈尔滨, 中国 \\ ayuqiang0708@163.com, bwzh7411@126.com
}

关键词：酒店企业; OTA; 竞争与竞合

中文摘要. 互联网时代背景下OTA借助信息技术, 实现了对传统旅游行业的逆袭。迫于OTA 网络渠道和消费体验创新的巨大优势, 传统旅游行业争相与OTA合作, 通过共享业务利润, 分享市场规模达到合作共赢的商业格局, 其中酒店行业就是最早与OTA谋求合作共赢的旅游 业产业分支之一。然而, 近年来, OTA行业竞争激烈发生着行业巨变, 出现了行业寡头, 使 得OTA的议价优势更为明显，同时，经过近10年的蛰伏，酒店企业在信息技术的引领下不断 实现行业创新和产业升级, 酒店企业的信息化创新能力不断增强, 因此, 酒店企业与OTA之 间重新出现了商业竞争和摩擦, 本文将围绕酒店企业如何发挥自身的创新优势在新一轮竞争 中与OTA实现竞争与竞合展开讨论与分析。

\section{1. 引言}

随着互联网时代的深入发展, OTA作为创新型的旅游电商发展模式, 凭借其平台的技术 优势拓宽了旅游客户的消费渠道, 提升了客户的消费体验, 酒店企业通过与OTA的合作, 能 够高效的获得订单, 极大的提升了客房的入住率, 具有比自营渠道或传统GDS营销更具竞争 力的价格优势。OTA为提升酒店企业的市场占有率和客户粘性都起到了积极的促进作用。然 
而, 近年来, OTA行业竞争的聚合度越来越高, 以Priceline、Expedia、Travelocity等为代表的 国外OTA平台以及携程、途牛和飞猪旅行为代表的国内OTA企业在激烈的市场竞争中逐渐突 出出来, 行业寡头的出现打破了以往合作中的平衡, 使得酒店企业为代表的传统旅游行业在 与OTA的市场议价方面逐渐失去了曾经的自信和主动, 酒店企业必须重新审视如何与OTA寡 头们的竞争与合作。

\section{2. 酒店企业与OTA的竞争}

\section{1 酒店企业在OTA平台的排位之争}

在OTA刚刚进入旅游市场的时候, 酒店企业纷纷主动与OTA平台合作, 为了能够在平台 上获得较为有利的排位, 不惜支付给平台更高的佣金甚至是价格的优惠来换取更高的排位或 更高的网络评级和分类, 而OTA平台也正是借助酒店企业们的慷慨解囊而获得了快速发展壮 大的难得机遇。然而, 随着OTA平台的逐渐整合, 在大浪淘沙中许多OTA平台要么被淘汰, 要么被收购, 最终只可能留下规模大影响力强的平台企业, 当前, 在中国的OTA行业中, 携 程、途牛和飞猪旅行等三家平台的客户访问量占线上旅游消费的 $70 \%$ 以上, 由于平台的流量 优势和应对激烈的市场竞争, 它们对酒店企业的入驻条件也越发苛刻起来, 很多OTA平台主 动要求酒店企业提高佣金比例以确保企业的平台排位能够继续保持优势, 这就势必造成酒店 企业的经营成本增加。在这轮排位之争中, 规模和品牌影响力大的企业反而是受影响最大的 企业, 因为, 品牌影响力大的企业对旅游消费者是有一定号召力和吸引力的, 能够让旅游消 费者选择入住大型酒店企业完全就是品牌效应的作用, 而现在, 由于OTA平台的佣金提高, 那些原本就会选择入住的客户现在酒店企业需要花费更高的代价才能从平台上获得订单, 交 易成本提高了但是却没有达到提升额外客户流量的目的。可见, 酒店企业与OTA平台的排位 之争早已打破合作之初时的相互默契和配合, OTA平台具有越来越大的话语权, 而酒店企业 为了控制经营成本也不可能轻易在佣金和价格上做过多的妥协, 因此, 新的商业矛盾和摩擦 在所难免。

\section{2 定价权之争}

早期OTA与酒店企业或是其他旅游行业企业合作时在终端销售价格上与企业的零售价格 是有高度默契和一致性的, 因为OTA平台仅仅是适应了旅游消费者线上消费体验的需求并不 能替代酒店企业等的线下服务, 在做线上代理的过程中定价协议一直是酒店企业们占主导地 位, 同时定价协议也是酒店企业约束OTA的有力工具避免出现市场价格战的乱象。然而, 多 年来, 由于旅游终端消费者逐渐形成了线上消费的惯性和习惯, OTA平台逐渐完全替代其他 任何形式旅游企业成为了消费者获取旅游信息的首选和唯一渠道, OTA们改变了消费者获取 价格信息的行为习惯和比价方式，过去消费者是参考酒店的渠道价格来参考平台销售价格， 现在消费者则是直接以线上OTA平台的销售价格作为唯一的价格参考标准, 酒店企业的自营 销售渠道的客户流量都被OTA平台所吸引, 而失去了客户流量的酒店官方平台的议价能力就 越来越弱, 渠道之争其实就是定价主导权之争, OTA平台们逐渐借助线上客户资源优势打破 了酒店企业定价权的主导地位, OTA平台为了更好的迎合消费者的线上消费需求，同时也是 为了与其他平台之间竞争中能够获得更大的优势, OTA平台对于线上终端消费定价权的控制 需求越来越强烈，价格一致性的平衡势必被打破了， OTA在利用平台流量优势打压酒店企业 定价机制的话语权, 它们开始主导价格竞争中的定价权, 导致的结果就是酒店企业的自营渠 道在价格上无法与线上OTA保持同步和默契, 在终端消费价格的议价能力上越来越被动, 甚 至在OTA的主动调价面前不得不被动跟进和响应, 酒店企业的线上预订价格在OTA平时上被 降价和被打折后, 提升的是OTA平台的市场竞争力和流量占有率, 然而, 牺牲的却是酒店企 业线下服务的运营成本, 压缩的却是酒店企业的经营利润, 可见, 在定价权之争中, OTA与 酒店企业的明争暗斗是难以调和的利益之争。 


\subsection{OTA与OTS服务理念之争}

最初，在旅游市场上出现OTA线上旅游代理是旅游行业为了适应信息时代背景下电子商 务模式创新的有益尝试的结果。电子商务的模式创新在旅游业发展应用的实践中选择了 OTA 线上代理的创新模式即是旅游行业发展的必然选择也是信息时代背景下旅游消费者的选择。 OTA平台成为了旅游产业链上全行业的信息平台和消费渠道, OTA们的代理经营降低了旅游 产业中包括酒店企业在内的所有旅游企业的经营成本，提升了经营效率，但是毕竟线上代理 的服务OTA们的优势和特长已经发挥到了极致, OTA的服务创新迫切寻求新的突破就必须从 最初的单一领域的价格代理和信息服务进行转变, OTA们在掌握了越来越多的旅游产业链数 据资源后结合客户流量的巨大优势, 开始尝试打包服务, 从旅游消费者计划出行的每一个环 节整合数据和信息资源, 利用其平台议价能力的优势, 整合旅游服务中吃、住、行、娱、乐 等全方位的价格套餐服务，这一理念的推出对旅游企业的市场冲击是极大的。

面对OTA平台的咄咄逼人，酒店企业为代表的传统旅游行业近年来也的探索着创新和改变。 一种基于线上平台实现旅游在线服务的OTS模式呈现在了人们眼前。与以电商为核心和主导 的OTA不同, OTS 是旅游企业基于自营电商平台和渠道打包和整合优质旅游服务提供给旅游 消费者的线上服务平台。当酒店企业跨界进入到电商领域并自己做起电商模式创新以后, 它 们对客户流量的吸引和拉动作用同样巨大, OTS的服务理念是基于传统旅游行业和标准化旅 游服务为核心展开的线上线下综合服务平台, 它与OTA只做线上代理不同, OTA追求的是传 统电商高效低成本的价格竞争优势, 可见, 酒店企业的OTS服务理念的出现和成熟彻底改变 了以往与OTA平台竞争的被动局面, 理念之争在未来一段较长时间内OTA与OTS的理念之争 会继续接受市场的检验。

\section{3. 酒店企业如何与OTA竞合}

\section{1 大数据分析的合作}

无论是电商主导的OTA平台还是酒店企业创新主导的OTS线上旅游服务平台, 在信息化 背景下的旅游创新服务都以追逐客户流量为重心。信息时代是信息产生价值的时代, 大数据 技术作为平台商业化运营的工具和手段离不开大数据的支撑和配合, 数据是信息的载体, 掌 握了数据就赢得了信息获取的主导权, 拥有了信息资源就具备了信息时代商业竞争的绝对优 势。在数据收集方面, 无论是OTA还是OTS都具备相当强的实力来实现终端消费客户基本信 息收集的功能，但是OTA只能收集到旅游消费者线上消费的相关信息却无法检测到线下消费 者消费体验的真实数据, 要想收集和获取消费者完整的旅游消费信息必须结合线下客户体验 的数据综合分析才能全面客观地分析旅游消费者的消费行为习惯以及消费偏好和心理特征。 因此, 为了满足信息化背景下市场营销对策的制定和实施, 大数据分析是不可或缺的技术保 障, 而大数据的来源需要酒店为代表的终端旅游服务提供企业配合OTA类的平台企业共同合 作才能获得真实全面的数据资源, 因此, 酒店企业是OTA大数据采集过程中不可或缺的重要 一环, OTS可以是OTA大数据分析的重要补充和参考。

\section{2 消费体验服务升级的合作}

随着信息时代步入到智能化的发展阶段, 酒店行业的信息化发展朝着智能化发展方向积 极迈进。智能化提升了旅游消费升级的进程, 我们可以看到越来越多的酒店企业将智能化的 设施、环境和技术应用到酒店企业的日常运营管理实践中, 从语音助手到客房智慧化管理, 从智能家居摆设陈列的设置到物联网技术的搭建, 现代酒店企业正在用智能信息领域的一切 技术手段来提升自己的服务能力改进酒店消费的服务场景, 用更加智慧化、安全化、个性化 的服务去吸引越来越挑剔的旅游消费者, OTA在这轮旅游消费升级的响应过程中必须审时度 势积极与酒店企业合作才能获得自己的一席之地, OTA掌握的客户流量资源要想能够在旅游 
消费升级中变现创造更大的商业价值，需要积极与传统旅游企业合作开发基于旅游终端消费 者行为习惯和需求的创新产品和服务, 可以说OTA竞争优势在线上, 酒店企业的竞争优势在 线下，只有将线上线下的服务整合才是未来响应旅游消费体验升级的唯一手段，通过整合线 上和线下的服务优势才能拟补双方各自的缺陷和不足，才能更好的携手并进合作共赢。

\section{4. 结束语}

传统电商主导下的OTA凭借平台的技术优势获得了对传统旅游行业的每个产业链节点的 竞争优势, OTA激发了酒店行业的自省和升级, 双方的合作也随着OTA市场的聚合式发展逐 渐打破了原有的战略平衡, 然而市场的有序竞争是促进行业发展满足旅游市场消费升级的内 在动力, 酒店企业与OTA之间的合作和竞争必然长期共存, 并在市场竞争中寻求合作与共赢, 未来行业发展的主导和引领其实不应该是传统旅游业也不是OTA为代表的电商平台，而应以 市场需求为导向, 发挥各自企业优势, 服务于旅游消费市场升级为核心, 并以此为前提展开 的竞争与竞合。

\section{致谢}

本文系作者在哈尔滨香坊永泰喜来登酒店挂职锻炼学习期间的学习成果之一。

\section{References}

[1] Bitran G R, Mondschein S V, An application of yield management to the hotel industry considering multiple day stays, Operations Research, vol.43( 3) ,pp.427-443, 1995.

[2] Buhalis D, Relationships in the distribution channel of tourism: conflicts between hoteliers and tour operators in the mediterranean region, International Journal of Hospitality \& Tourism Administration, vol.1, pp. 113-119, 2000.

[3] El -Haber S, El-Taha M, Dynamic two-leg airline seat inventory control with overbooking, cancellations and no-shows, Journal of Revenue and Pricing Management, vol.3, pp. 143-150, 2004.

[4] DeKay F, Yates B, Toh R S, Non-performance penalties in the hotel industry, International Journal of Hospitality Management, vol. 23, pp. 273-286, 2004.

[5] Cakanyildirim M, Feng Q, Gan X H, Sethi S P, Contracting and coordination under asymmetric production cost information, Production and Operation Management, vol. 21, pp. 345-360, 2012.

[6] Lee H, Guillet B D, Law R, An Examination of the Relationship between Online Travel Agents and Hotels A Case Study of Choice Hotels International and Expedia.com, Cornell Hospitality Quarterly, vol. 54, pp. 95-107,2013.

[7] Riadh Ladhari, Mélissa Michaud, eWOM effects on hotel booking intentions,attitudes, trust, and website perceptions, International Journal of HospitalityManagement, vol.46, pp.36-45, 2015.

[8] Paul Phillips, Krystin Zigan, Maria Manuela Santos Silva, Roland Schegg, The interactive effects of online reviews on the determinants of Swiss hotel performance: A neural network analysis, Tourism Management, vol. 50, pp. 130-141, 2016. 